\title{
Modelo geometalúrgico de la caracterización de arcillas para la eficiencia de la flotación usando la metodología de machine learning
}

\author{
Geometallurgical model of the characterization of clays for flotation efficiency \\ using machine learning methodology
}

Julio Alejandro Castro Andrade ${ }^{1}$, Julia Marilú Calderón Celis²

Recibido: 16/12/2020 - Aprobado: 17/09/2021 - Publicado: 23/12/2021

\begin{abstract}
RESUMEN
El modelo geometalúrgico es el resultado de la integración de las disciplinas de geología, minería y metalurgia con la finalidad de agregar valor y disminuiría el riesgo en un proceso como por ejemplo la eficiencia de flotación de calcopirita. El objetivo de este estudio fue calibrar modelos para cuantificar el contenido de arcillas y gangas de un yacimiento hidrotermal del tipo pórfido cobre-oro por espectroscopía NIR. La metodología seleccionada fue diseñar un modelo quimiométrico basado en 173 compositos de taladros diamantinos a los que se le realizó ensayos de difracción de rayos x y espectroscopia de infrarrojo cercano, validándose los resultados con una validación cruzada a través de una metodología machine learning. La creación de los modelos se realizó mediante una regresión no lineal regularizada por el método de Ridge. Se obtuvo modelos con baja linealidad para minerales de calcita y plagioclasa, con valores de R2 ( 0.51 y 0.78 , respectivamente). El modelo de regresión presenta una linealidad para minerales de esmectita, cuarzo, ortoclasa y muscovita mostró un alto $\mathrm{R} 2(0.95,0.93,0.64$ y 0.59 , respectivamente). Los resultados encontrados para el contenido de arcillas y gangas indican que los análisis de difracción de rayos x se pueden sustituir, en gran parte, por los modelos espectrales. En el caso de la calcita y plagioclasa, sería conveniente realizar una campaña de caracterización, con la finalidad de mejorar el modelo y poder sustituir los análisis de difracción de rayos x para estas especies, lo que permitirá generar un modelo geometalúrgico de una forma rápida y eficiente con un método semi cuantitativo.
\end{abstract}

Palabras claves: Mineralogía; difracción de rayos x; espectroscopia del infrarrojo cercano; quimiometría; machine learning; validación cruzada; flotación.

\begin{abstract}
The geometallurgical model is the result of the integration of the disciplines of geology, mining and metallurgy in order to add value and reduce risk in a process such as the flotation efficiency of chalcopyrite. The objective of this study was to calibrate models to quantify the content of clays and gangas of a hydrothermal deposit of the porphyry copper-gold type by NIR spectroscopy. The selected methodology was to design a chemometric model based on 173 diamond drill composites to which X-ray diffraction tests and near infrared spectroscopy were performed, validating the results with a cross-validation through a machine learning methodology. The creation of the models was carried out by means of a regularized non-linear regression by the Ridge method. Low linearity models were obtained for calcite and plagioclase minerals, with R2 values ( 0.51 and 0.78 , respectively). The regression model presents a linearity for smectite, quartz, orthoclase and muscovite minerals showed a high R2 $(0.95,0.93,0.64$ and 0.59 , respectively). The results found for the content of clays and gangue indicate that $X$-ray diffraction analyzes can be largely replaced by spectral models. In the case of calcite and plagioclase, it would be convenient to carry out a characterization campaign, in order to improve the model and to be able to replace the x-ray diffraction analyzes for these species, which will allow to generate a geometallurgical model in a quick and easy way. efficient with a semi-quantitative method.
\end{abstract}

Keywords: Mineralogy; x-ray diffraction; near infrared spectroscopy; chemometrics; machine learning; cross validation; froth flotation.

\footnotetext{
1 Universidad Nacional Mayor de San Marcos,Unidad de Posgrado, Lima, Perú. Ingeniero Metalúrgico, Maestría Geometalurgia. Autor para correspondencia: juio.castro7@unmsm.edu.pe - ORCID: https://orcid.org/0000-0003-0507-6680

2 Universidad Nacional Mayor de San Marcos, Lima, Perú. Ingeniería Industrial. Asesora de Tesis de Pregrado y Posgrado.

E-mail: jcalderond2@unmsm.edu.pe / calderonmarilu@gmail.com - ORCID: https://orcid.org/0000-0002-1374-9307
} 


\section{INTRODUCCIÓN}

El presente trabajo de investigación pretendió obtener valores cuantitativos del contenido de arcillas a través de modelos quimiométricos aplicados a datos obtenidos de mediciones espectroscópicas en el rango infrarrojo cercano. Para realizar este trabajo se utilizó como datos las campañas de sondaje para caracterización mineralógica con ensayos de difracción de rayos x. Este trabajo se desarrolló en dos etapas, la primera consistió en la consolidación de las bases de datos y la definición de minerales a modelar, mientras que en la segunda se desarrolló y evaluó los modelos (Vilches Nuñez, 2018).

Las variables mineralógicas criticas afectan sensiblemente el proceso de flotación, este es el caso de la presencia de arcillas. Su ocurrencia de estas gangas es variable en cada tipo de yacimiento por los que es importante modelar su comportamiento banco a banco según el avance de minado y de esta manera implementar un modelo geometalúrgico (Canchaya Moya, 2008).
La problemática de modelar los espectros del infrarrojo cercano es que se recoge una gran cantidad de datos con diferentes características de alta dimensionalidad que no pueden ser manejables de manera tradicional. Anaconda Navigator es una interfaz gráfica de usuario bastante sencilla, pero con un potencial enorme. Puede gestionar de manera avanzada paquetes relacionados a la ciencia de datos con Python lo cual ayuda a realizar modelos geometalúrgicos a partir de un gran volumen de datos.

\section{MÉTODOS}

\subsection{Materiales}

\subsubsection{Consolidación de la base de datos}

Cada arcilla tiene una distribución espacial correspondiente según el taladro de perforación diamantina, en la Figura 1 se puede observar la ocurrencia de las principales arcillas en el yacimiento del tipo pórfido cobre - oro.

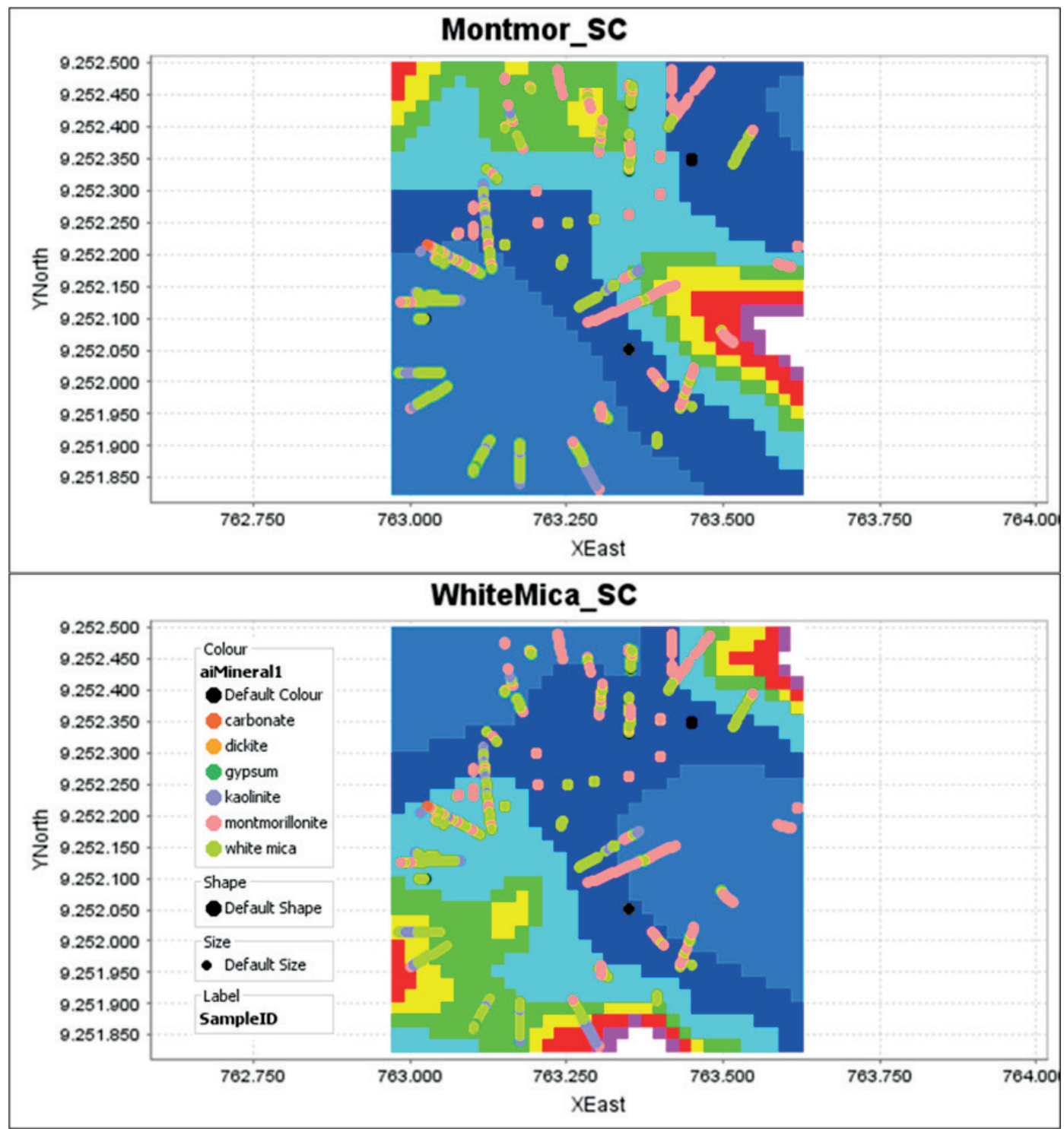

Figura 1. Distribución espacial de las principales arcillas determinado por aiSiris y coordenadas de toma de muestra por perforación diamantina. 
Para poder uniformar la base de datos se consideraron los siguientes grupos de minerales (Tabla 1):

Tabla 1. Agrupaciones minerales que serán utilizadas como base en la construcción de modelos quimiométricos

\begin{tabular}{llc}
\hline ID & Muestras & Porcentaje \\
\hline 1 & montmorillonite+chlorite & $35.53 \%$ \\
2 & white mica+Quartz & $25.82 \%$ \\
3 & white mica+hematite & $7.69 \%$ \\
4 & kaolinite+Quartz & $7.33 \%$ \\
5 & montmorillonite+Quartz & $7.33 \%$ \\
6 & white mica+chlorite & $6.96 \%$ \\
7 & montmorillonite+hematite & $4.03 \%$ \\
8 & white mica+goethite+hematite & $1.10 \%$ \\
9 & white mica+jarosite & $0.92 \%$ \\
10 & white mica+chlorite+hematite & $0.55 \%$ \\
11 & montmorillonite+chlorite+hematite & $0.55 \%$ \\
12 & white mica+goethite & $0.37 \%$ \\
13 & kaolinite+hematite & $0.37 \%$ \\
14 & kaolinite+chlorite & $0.37 \%$ \\
15 & montmorillonite+goethite+hematite & $0.37 \%$ \\
16 & carbonate+Quartz & $0.18 \%$ \\
17 & dickite+Quartz & $0.18 \%$ \\
18 & kaolinite+goethite & $0.18 \%$ \\
19 & gypsum+Quartz & $0.18 \%$ \\
\hline
\end{tabular}

Una vez consolidada la información se procede a realizar modelos preliminares con el objetivo de definir cuáles minerales podrían ser mejor modelados, para este fin se utilizó metodología de validación cruzada y se evaluará el indicador $\mathrm{R}^{2}$, los resultados se resumen en la Figura 2.

\subsection{Métodos}

Como herramienta matemática a utilizar para la construcción de los modelos quimiométricos se seleccionó el estándar del mercado, es decir, al algoritmo RIDGE, siendo Anaconda Navigator el software seleccionado para su implementación.

Para el algoritmo RIDGE se usó como variable independiente el espectro NIR y como variable objetivo se definió los minerales y el número de componentes principales para utilizar para la resolución del problema; en este caso el software a utilizar genera entrenamientos con distintos números de componentes y entrega un gráfico de correlación, facilitando la definición del número de componentes a utilizar.

La metodología seleccionada para la construcción de los modelos quimiométricos es una metodología que se ha utilizado en varios yacimientos en la minería mundial y corresponde a una validación cruzada. Para explicar en qué consiste la validación cruzada nos apoyaremos en la Figura 3. Existe un universo de datos asociado a la data de entrenamiento (rectángulo verde), el cual es dividido en 10 grupos aleatorios de similar cantidad de datos (rectángulos plomos, desde G-1 a G-10).

Este procedimiento involucra construir 10 modelos, uno por cada etapa de validación, en la Figura 3 se representan las etapas de validación con una $\mathrm{V}$, es decir estas etapas van desde la V1 a la V10. En cada etapa de validación se genera un set de entrenamiento constituido por 9 grupos, en el caso de V1 se entrena un modelo con los grupos que van de G-2 a G-10, luego se evalúa la data existente en G-1 para generar el bloque E-1 representado en rojo, el bloque E-1 contiene la evaluación del modelo

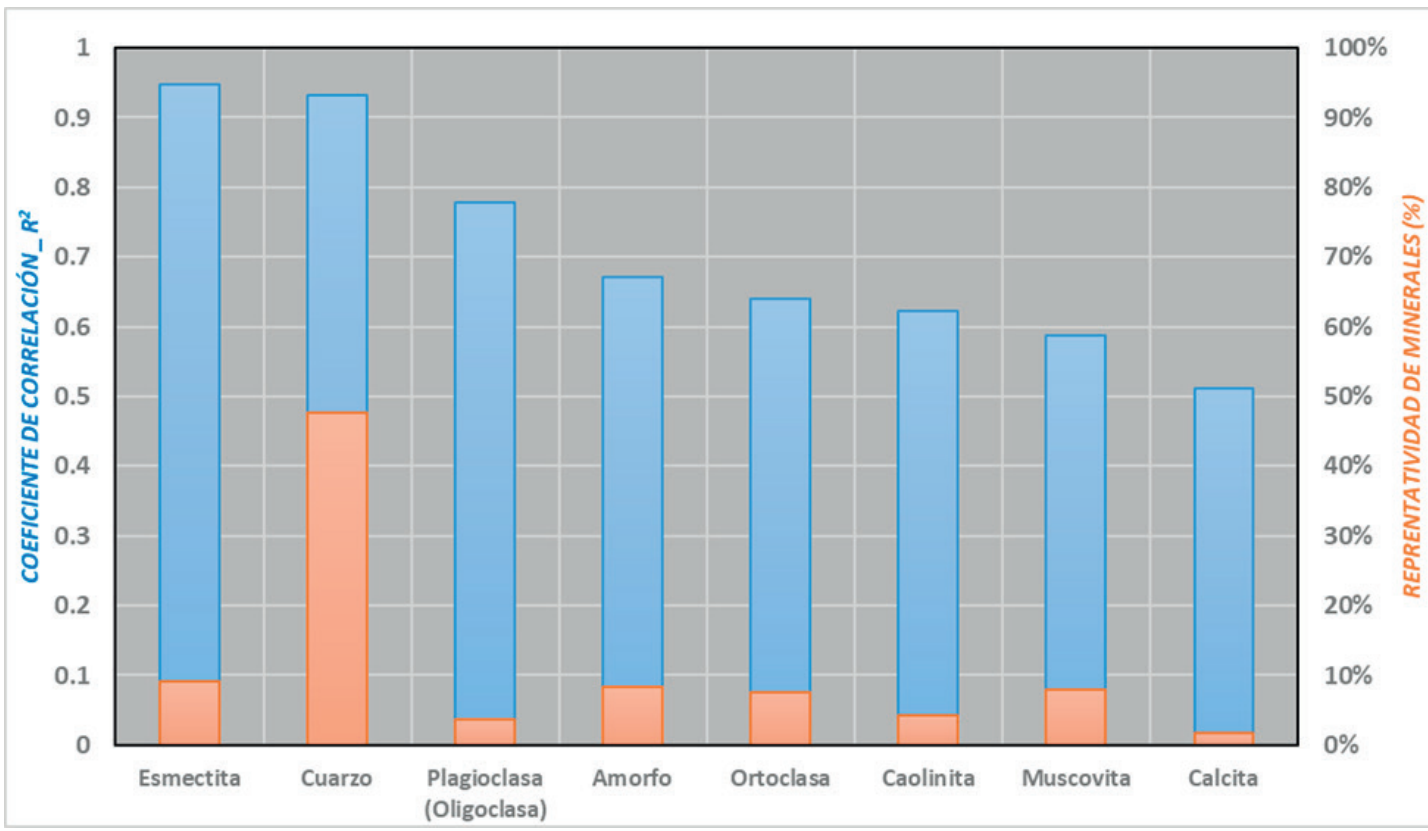

Figura 2. Se muestra en barras azules (eje izquierdo) el $\mathrm{R}^{2}$ obtenido por validación cruzada en modelos quimiométricos preliminares, y en barras naranjas (eje derecho) el contenido promedio de los minerales en las muestras analizadas se puede observar que los minerales más abundantes son el Cuarzo, Esmectita, Muscovita, Amorfo y Ortoclasa. 
construido en V-1 en la data existente en G-1. Este procedimiento se repite 10 veces hasta haber evaluado la data de entrenamiento en su totalidad.

En este punto es posible comparar el resultado de la validación cruzada al comparar el conjunto de datos evaluados con la data original de entrenamiento como se observa en la Figura 4.

Los indicadores generados en este punto son adecuados para evaluar la calidad del modelo, y si se observan resultados adecuados es posible construir un modelo único con toda la data de entrenamiento, ese será el modelo oficial para entregar.

\subsection{Ridge regression}

Según Pallares Cabrera (2014), la técnica Ridge Regresión (RR) se basa en un modelo de regresión que tiene como objetivo minimizar el error evitando el sobreajuste, basándose en términos de penalizaciones (w). El modelo de Ridge se define con la siguiente ecuación.

$$
F(w)=k\|w\|^{2}+\sum\left(y_{i}-X_{i} w\right)^{2} \quad \ldots \text { Ecuación } 01
$$

Donde k es el coeficiente Ridge, al derivar la función y despejar w:

$$
w=\left(X^{T} X+k l_{n}\right)^{-1} X^{T} y \quad \ldots \text { Ecuación } 02
$$

La predicción puede ser escrita como

$$
\hat{y}(x)=x w=\sum_{i=1}^{n} w_{i}(x)_{i} \quad \ldots \text { Ecuación } 03
$$

\subsection{Selección y entrenamiento del modelo \\ 2.4.1. Error de validación}

La validación de un modelo de regresión no lineal depende de su generalización, que se traduce en tener una buena predicción de resultados al ingresar nuevos valores.

Para generar un modelo confiable usando machine learning, se debe considerar subconjuntos de datos de entrenamiento y prueba. La finalidad de tener sub conjunto de datos es determinar el error de la estimación sin necesidad de realizar un sobre ajuste (overfitting) o sub ajuste (underfitting).

En la Figura 5 se demuestra la tendencia de valores asintóticos de los errores de perdición y como forzar un modelo me genera un sobre ajuste.

\subsubsection{Varianza}

La varianza de datos, es una medida de dispersión respecto de la media. Cuando este valor aumenta indica un sesgo considerable de los valores en una población de datos.

Para determinar una suma de errores es recomendable

\begin{tabular}{|c|c|c|c|c|c|c|c|c|c|c|}
\hline $\mathrm{G} 01$ & E01 & G01 & G01 & G01 & $\mathrm{G} 01$ & G01 & G01 & G01 & G01 & G01 \\
\hline $\mathrm{GO2}$ & G02 & E02 & G02 & $\mathrm{G} 02$ & $\mathrm{GO2}$ & $\mathrm{G} 02$ & $\mathrm{GO2}$ & $\mathrm{GO2}$ & $\mathrm{GO2}$ & $\mathrm{GO} 2$ \\
\hline $\mathrm{G} 03$ & G03 & $\mathrm{GO3}$ & $\mathrm{E} 03$ & $\mathrm{G03}$ & $\mathrm{G} 03$ & G03 & $\mathrm{G} 03$ & G03 & $\mathrm{G03}$ & $\mathrm{GO3}$ \\
\hline G04 & G04 & G04 & G04 & E04 & G04 & G04 & G04 & G04 & $\mathrm{GO4}$ & G04 \\
\hline G05 & G05 & G05 & G05 & G05 & E05 & G05 & G05 & G05 & G05 & G05 \\
\hline G06 & G06 & G06 & G06 & G06 & G06 & E06 & G06 & G06 & G06 & G06 \\
\hline G07 & G07 & G07 & G07 & G07 & G07 & G07 & E07 & G07 & G07 & G07 \\
\hline G08 & G08 & G08 & G08 & G08 & G08 & G08 & G08 & E08 & G08 & G08 \\
\hline $\mathrm{G} 09$ & G09 & G09 & $\mathrm{G} 09$ & G09 & $\mathrm{G} 09$ & G09 & G09 & G09 & E09 & G09 \\
\hline \multirow[t]{2}{*}{ G10 } & G10 & G10 & G10 & G10 & G10 & G10 & G10 & G10 & G10 & E10 \\
\hline & V01 & V02 & V03 & V04 & V05 & V06 & V07 & V08 & V09 & V10 \\
\hline
\end{tabular}
usar este estadístico de dispersión para demostrar la aditividad de los mismos.

Figura 3. Metodología de validación cruzada.

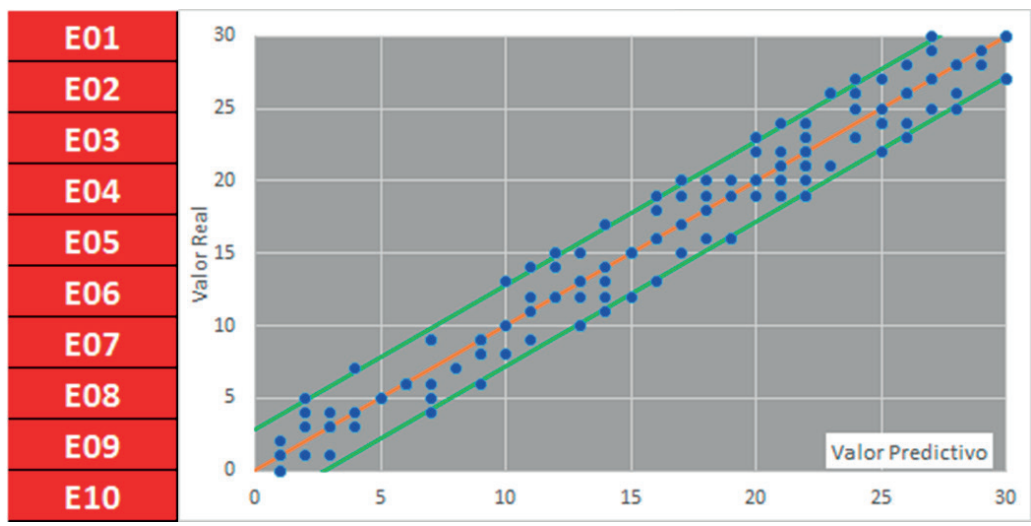

Figura 4. Resultado del proceso de validación cruzada. 


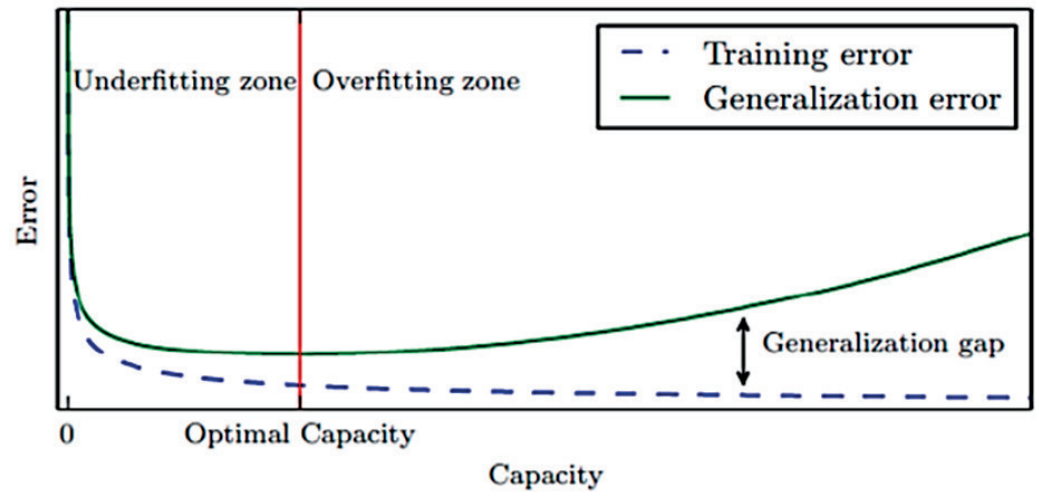

Figura 5. Análisis gráfico del underfitting y overfitting (González-Páramo, 2017)

Este valor se calcula restando los valores cuadráticos de cada registro respecto de su media para una población de datos.

$$
\begin{aligned}
S_{X}^{2}= & \frac{\left(X_{i}-\bar{X}\right)^{2}}{n-1}=\frac{1}{n-1} \sum_{i=1}^{n}\left(X_{i}-\bar{X}\right)^{2} \ldots \text { Ecuación } 04 \\
& \text { 2.4.3. Error cuadrático y desviación }
\end{aligned}
$$

El error cuadrático mide el promedio de los errores de los registros al cuadrado y es representado por las siglas MSE.

$\operatorname{MSE}(\bar{X})=E\left((\bar{X}-u)^{2}\right)=\left(\frac{\sigma}{\sqrt{n}}\right)^{2}=\frac{\sigma^{2}}{n}$ .. Ecuación 05

La desviación de este estimador (RMSE), nos ayuda a comparar en unidades adimensionales el error presente entre un valor predicho y real. Su calculo se realiza sacando la raíz cuadrada del error cuadrático.

$$
\operatorname{RMSE}(\bar{X})=\sqrt{\frac{1}{n} * \sum_{i=1}^{n}\left(X_{i}-\bar{X}\right)^{2}} \quad \ldots \text { Ecuación } 06
$$

\subsection{Minerales de arcillas}

Los minerales arcillosos provienen de la hidratación de los filosilicatos de aluminio, que según su formación pueden contener tierras alcalinas, magnesio y hierro (Pallares Cabrera, 2014).

Su procedencia es a través de la meteorización de las rocas ígneas de granito erosionadas como se observa en la Figura 6. Su coloración depende del tipo de impurezas que contiene que puede ir desde un blanco hasta anaranjado.

En la flotación de espumas existen varios grupos de filosilicatos que afectan de manera significativa en los procesos metalúrgicos, como son las esmectitas, caolinitas e illitas. La composición química de estas principales arcillas se observa en la Tabla 2.

Debido a su reología de pulpa, las arcillas se caracterizan principalmente por ser higroscópicas lo cual afecta al procesamiento metalúrgico por su alto consumo de reactivos y contener partículas microscópicas hasta de $10 \mathrm{um}$.

\subsection{Caracterización del mineral}

\subsubsection{Difracción de rayos $x$}

Comprender la mineralogía masiva de la muestra y el depósito en sí es un paso necesario. La difracción de rayos $\mathrm{X}$ (XRD) es un método analítico versátil para analizar las propiedades del material, como la identificación de fases cristalinas y su proporción relativa de una muestra de material particulado. La identificación de las fases se logra comparando el patrón de difracción de rayos $\mathrm{X}$ (Figura 7) obtenido de la muestra que se está midiendo con una base de datos de referencia. Con una sola medición, se pueden identificar fases minerales (cualitativas) y sus concentraciones / proporciones (cuantitativas). Los rayos $\mathrm{X}$ se generan en un difractómetro de laboratorio utilizando tubos de rayos $\mathrm{X}$ con un material de ánodo adecuado $(\mathrm{Cu}$, $\mathrm{Fe}$, etc.).

El difractómetro utilizado es D8 Quest con un goniómetro de geometría Kappa de 4 círculos, detector CMOS 2D Photon 100 shutterless, tubo de Rayos-X de molibdeno y monocromador de grafito TRIUMPH. Provisto de un criostato de rutina Oxford Cryosystems Desktop Cooler (170-290K) sin requerimiento de nitrógeno líquido y un cristato Bruker Kryoflex II para medidas entre 80-400K.

\subsubsection{Espectroscopia nir}

La espectroscopia de infrarrojo cercano (NIR) es un método utilizado en la determinación de sedimentos fluviales de cuencas de ríos, pudiendo ser de un uso extrapolado para caracterizar arcillas debido a su rango de espectro de 700 a 2500 nanómetros (Figura 8).

Equipos como el Terraspec 04 a permitido poder ampliar el rango de espectros con la finalidad de trabajar con minerales que anteriormente eran difícil de lecturar, como es el caso de las arcillas blancas, de esta manera el rango para estos equipos a pasado a ser de $350 \mathrm{~nm}$ hasta $2500 \mathrm{~nm}$, permitiendo de realizar una semi cuantificación del contenido de las principales arcillas.

En el presente trabajo se usó el equipo Terraspec 04 como se muestra en la Figura 9, el cual es un analizador de minerales que proporciona análisis portátiles, rápidos y no destructivos en el laboratorio o en el campo. 


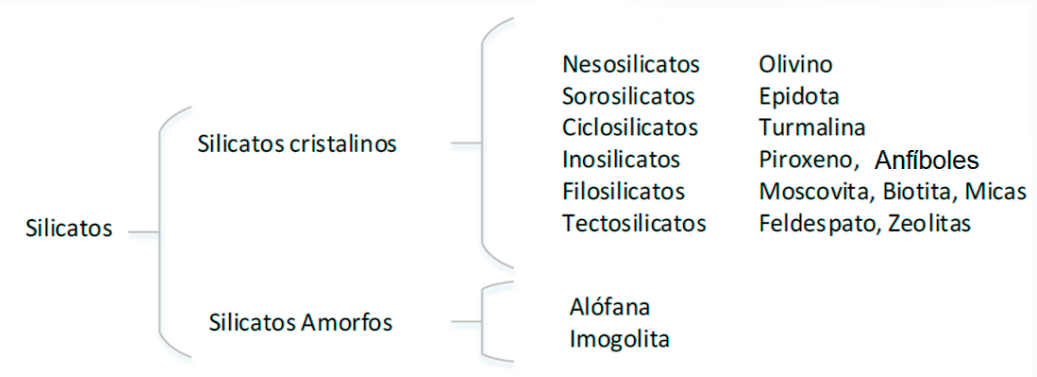

Figura 6. Mapa semántico de la subdivisión de minerales silicatados (Vilches Nuñez, 2018)

Tabla 2. Clasificación de las principales arcillas

\begin{tabular}{|c|c|c|}
\hline Grupo & Mineral & Formula \\
\hline \multirow[t]{5}{*}{ Grupo Caolín } & Caolinita & $\mathrm{Al} 2 \mathrm{O} 3 * 2 \mathrm{SiO} 2 * 2 \mathrm{H} 2 \mathrm{O}$ \\
\hline & Dickita & $\mathrm{Al} 2 \mathrm{O} 3 * 2 \mathrm{SiO} 2 * 2 \mathrm{H} 2 \mathrm{O}$ \\
\hline & Nacrita & $\mathrm{Al} 2 \mathrm{O} 3 * 2 \mathrm{SiO} 2 * 2 \mathrm{H} 2 \mathrm{O}$ \\
\hline & Halloysita & $(\mathrm{OH}) 3 \mathrm{Si} 4 \mathrm{Al} 4 \mathrm{O} 10 * \mathrm{H} 2 \mathrm{O}$ \\
\hline & Halloysita deshidratada & $(\mathrm{OH}) 5 \mathrm{Si} 4 \mathrm{Al} 4010$ \\
\hline Grupo de las Smectitas & Montmorillonita & $(\mathrm{Na}, \mathrm{Ca}) \mathrm{OAl} 2 \mathrm{O} 3 * 5 \mathrm{SiO} 2 * n \mathrm{H} 2 \mathrm{O}$ \\
\hline \multirow[t]{4}{*}{ (Montmorillonitas) } & Saponita & $2 \mathrm{MgO} * \mathrm{SiO} 2 * n H 2 O$ \\
\hline & Nontronita & $(\mathrm{Al}, \mathrm{Fe}) \mathrm{O} 3 * 3 \mathrm{SiO} 2 * n \mathrm{H} 2 \mathrm{O}$ \\
\hline & Beidelita & $\mathrm{Al} 4 \mathrm{O} 10 * 3 \mathrm{SiO} 2 * n \mathrm{H} 2 \mathrm{O}$ \\
\hline & Hectorita & $(\mathrm{Mg}, \mathrm{Li}) 3 * \mathrm{Si} 4 \mathrm{O} 2(\mathrm{OH}) 2 * \mathrm{H} 2 \mathrm{O}$ \\
\hline Grupo de las Illitas & Illita & $3 \mathrm{Al} 2 \mathrm{O} 3 * 6 \mathrm{SiO} 2 * \mathrm{~K} 2 \mathrm{O}$ \\
\hline \multirow[t]{2}{*}{ Grupo de las Hormitas } & Paligorskita & $2 \mathrm{MgO} * 3 \mathrm{SiO} 2 * 4 \mathrm{H} 2 \mathrm{O}$ \\
\hline & Sepiolita & $\mathrm{Mg} 4(\mathrm{OH}) 2 \mathrm{Si} 6013 * 6 \mathrm{H} 2 \mathrm{O}$ \\
\hline
\end{tabular}

Fuente: Influencia de arcillas en la flotación de material calcopiritico, proveniente de Codelco división Andina franco (Vilches Nuñez, 2018).
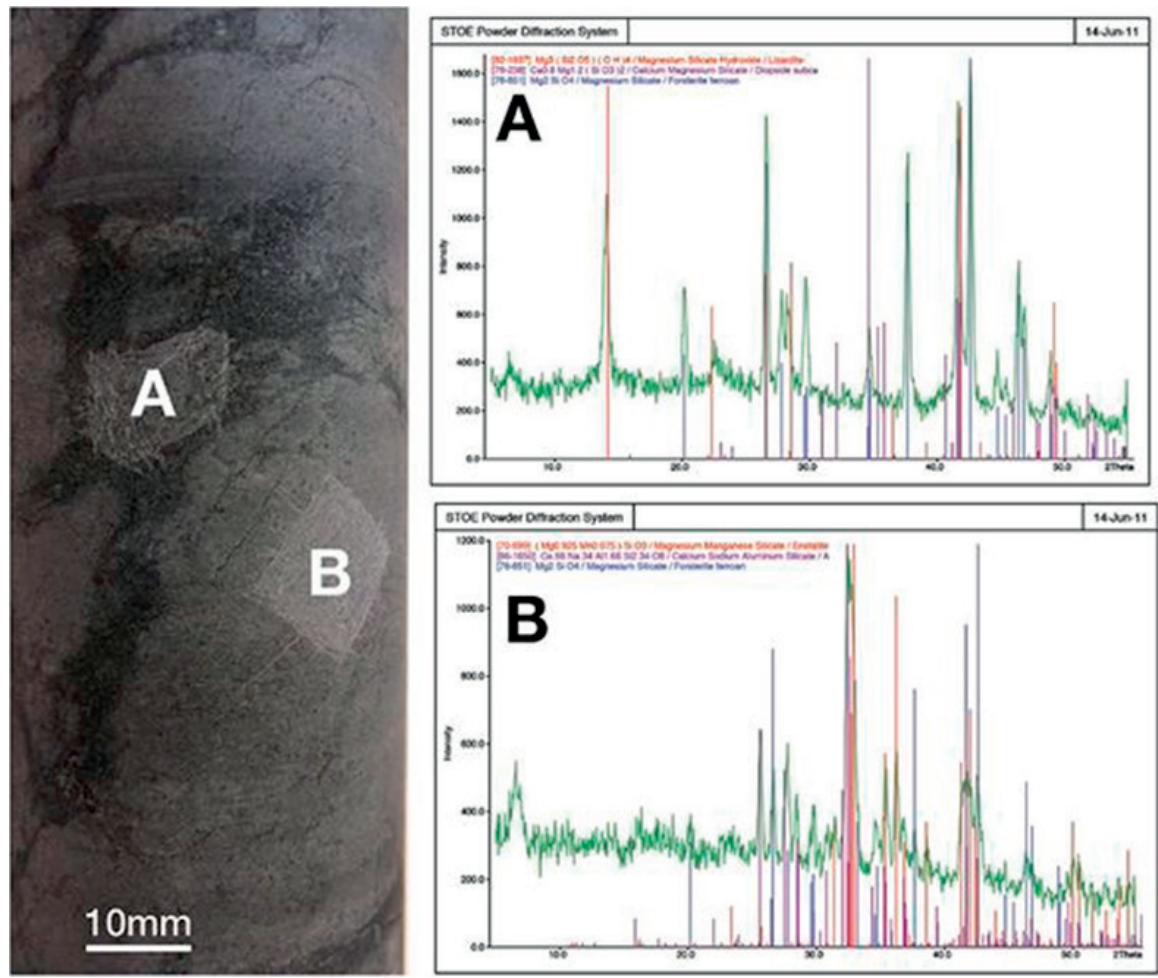

Figura 7. Acercamiento de una litología gris clara de grano fino (B) entremezclada con una unidad más oscura, ultramórfica y magmática $(A)$ y las identificaciones de los minerales realizados por el analizados TERRA XRD (Coleman, 2000). 


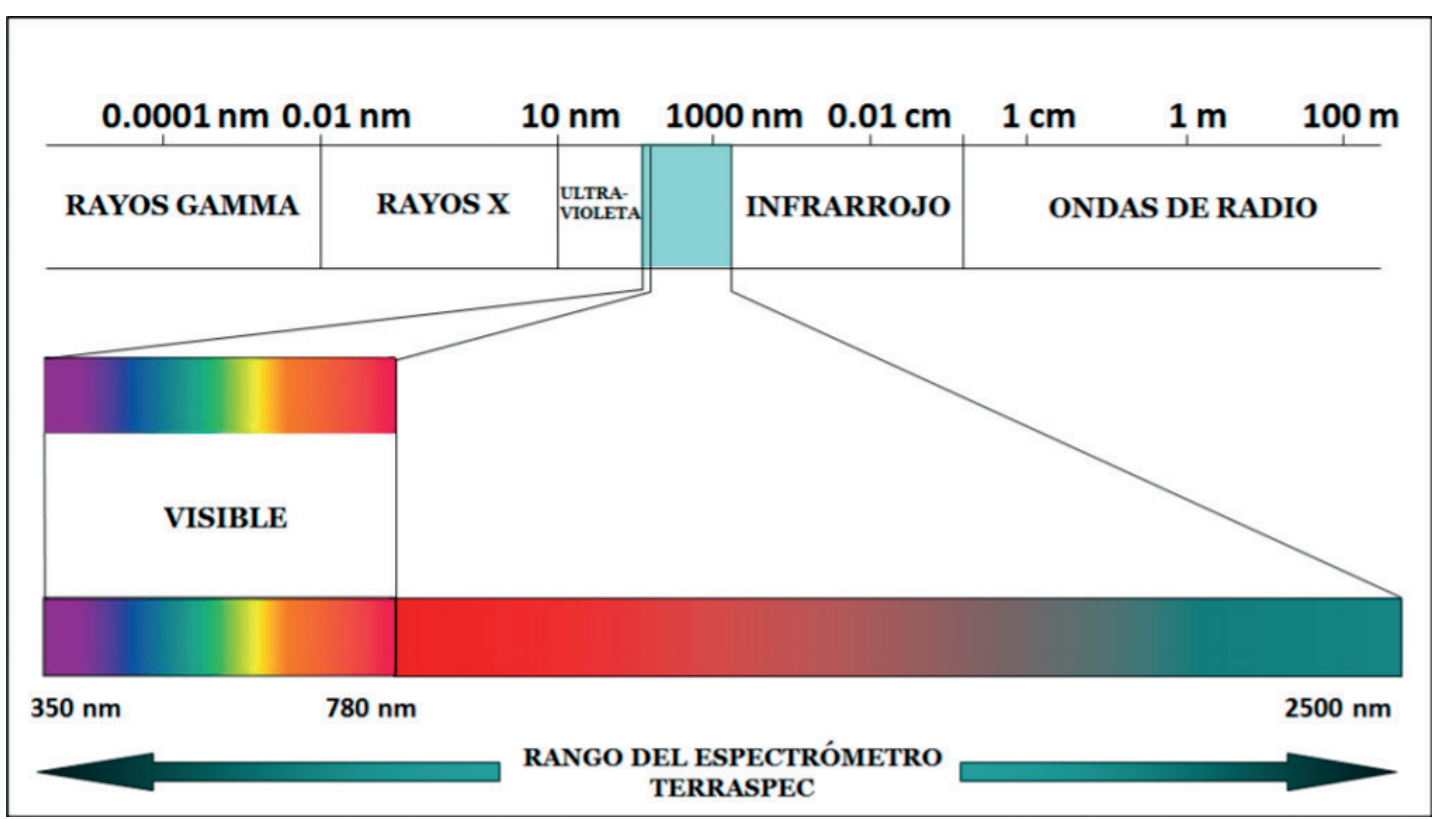

Figura 8. Espectro electromagnético y rango de medición del espectrómetro empleado en el estudio (Gómez Sánchez, 2015).

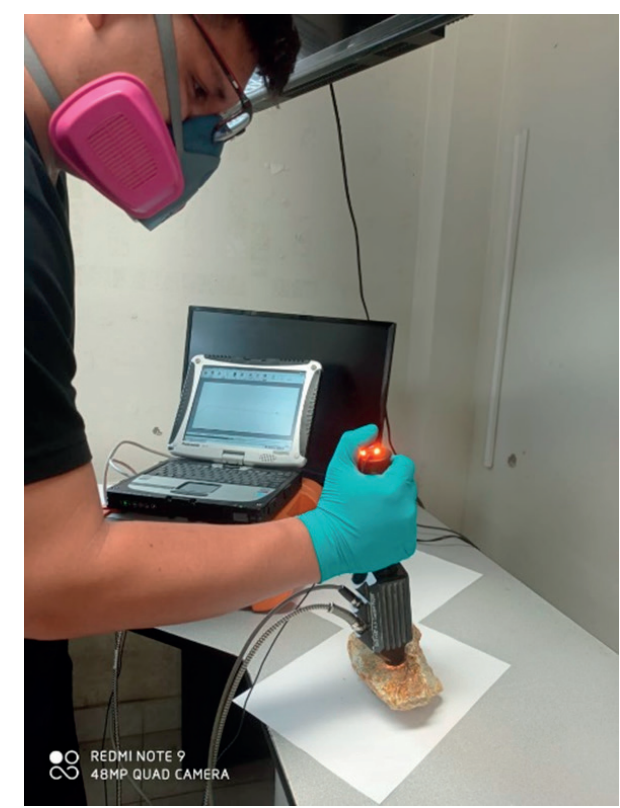

Figura 9. Lectura de muestra de mano con el equipo Terraspec 04 para la determinación del espectro de infrarrojo cercan

\section{RESULTADOS}

Para el análisis de datos se tiene una población de 139 valores de compositos para entrenar el modelo y 34 valores de compositos para validarlo tomados de una campaña de exploración por sondajes diamantinos en un pórfido de cobre-oro. A estos se les realizó análisis infrarrojo cercano con el equipo Terraspec 04 para el comparativo correspondiente (Figura10).

- Para el modelamiento de las especies representativas del pórfido, se realizó un análisis de ocurrencia como se muestra en la Figura11.
- En la Tabla 3, se observa la estadística descriptiva de los resultados determinados por difracción de rayos $\mathrm{x}$.

- Como se observa en la Tabla 4, se tiene un resumen de los indicadores para todos los minerales modelados:

- Se podría resumir que los modelos se podrían clasificar en 3 grupos:

- Excelente: Esmectita, Cuarzo, Ortoclasa y Muscovita. 


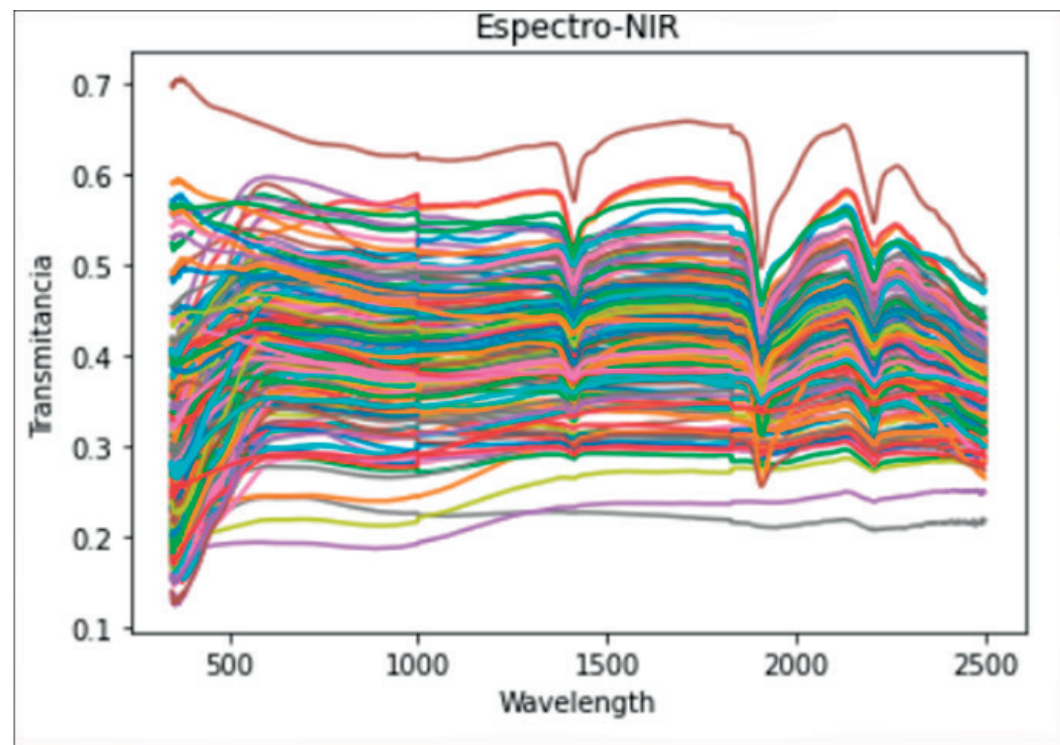

Figura 10. Gráfica de transmitancia por espectro para 173 compositos.
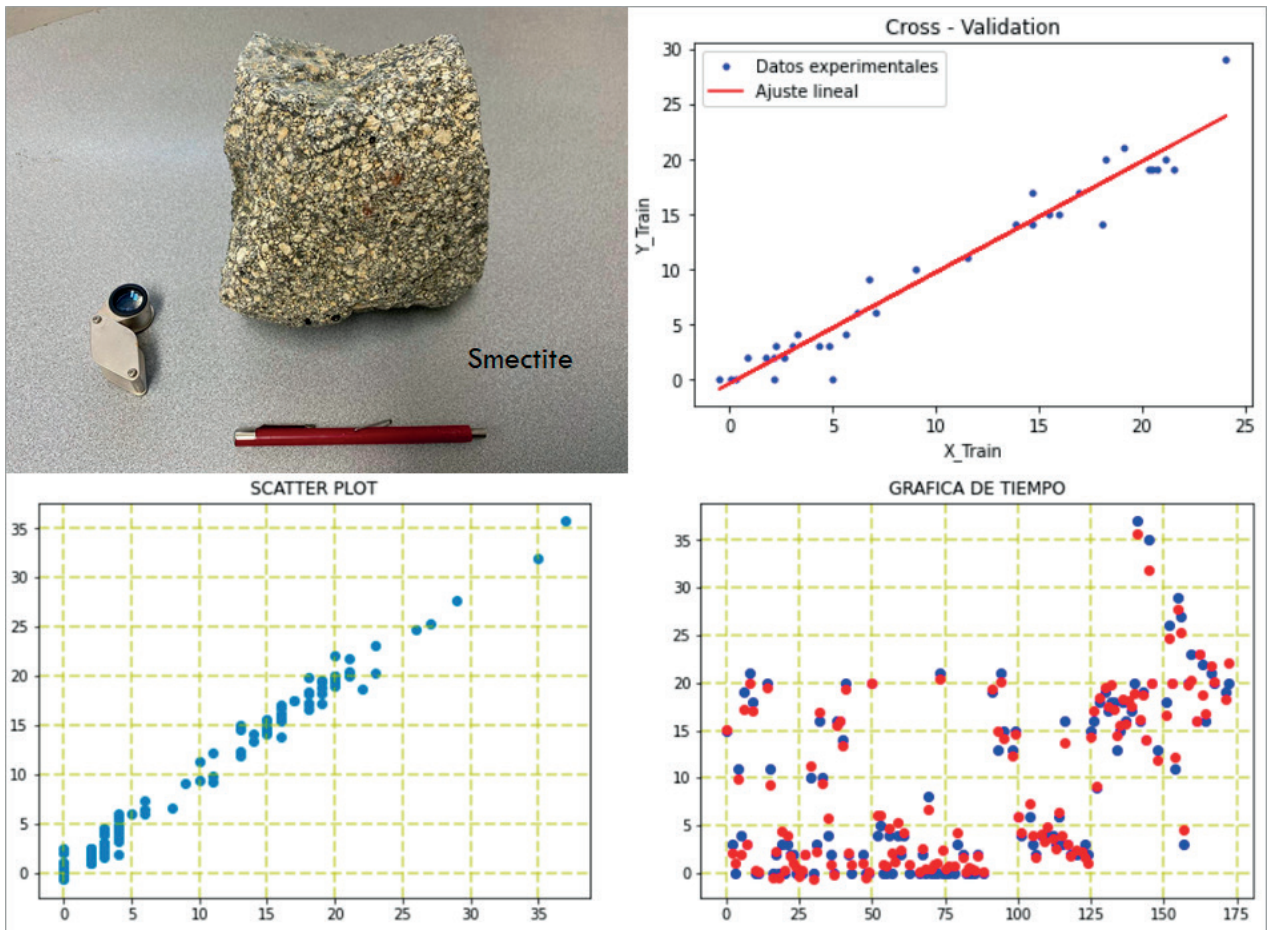

Figura 11. Análisis de ocurrencia para determinar las principales gangas a modelar.

Tabla 3. Distribución estadística de las especies modelas

\begin{tabular}{crrrrrrrr}
\hline & \multicolumn{1}{c}{ Calcita } & \multicolumn{1}{c}{ Caolinita } & \multicolumn{1}{c}{ Muscovita } & Plagioclasa (Oligoclasa) & \multicolumn{1}{c}{ Ortoclasa } & \multicolumn{1}{c}{ Cuarzo } & \multicolumn{1}{c}{ Amorfo } & Esmectita \\
\hline count & 173.0000000 & 173.0000000 & 173.0000000 & 173.0000000 & 173.0000000 & 173.0000000 & 173.0000000 & 173.00000000 \\
mean & 1.5000000 & 4.2196530 & 8.0895950 & 2.8526010 & 7.5549130 & 48.0809250 & 8.4508670 & 9.1849710 \\
std & 2.2457970 & 3.2686430 & 3.6207260 & 4.5513810 & 4.6294230 & 15.6065110 & 6.2760310 & 8.7244910 \\
min & 0.0000000 & 0.0000000 & 0.0000000 & 0.0000000 & 0.0000000 & 17.00000000 & 2.0000000 & 0.00000000 \\
$\mathbf{2 5 \%}$ & 0.0000000 & 2.0000000 & 5.0000000 & 0.0000000 & 4.0000000 & 37.0000000 & 4.0000000 & 2.0000000 \\
$\mathbf{5 0 \%}$ & 0.0000000 & 3.0000000 & 8.0000000 & 0.0000000 & 8.0000000 & 46.0000000 & 5.0000000 & 4.00000000 \\
$\mathbf{7 5 \%}$ & 3.0000000 & 6.0000000 & 10.0000000 & 5.0000000 & 11.0000000 & 59.0000000 & 14.0000000 & 17.00000000 \\
$\max$ & 7.5000000 & 12.0000000 & 17.5000000 & 12.5000000 & 21.5000000 & 86.00000000 & 25.0000000 & 37.00000000 \\
\hline
\end{tabular}


- Bueno: Caolinita y Amorfo.

- Regular: Calcita, Plagioclasa.

\subsection{Esmectita}

En el modelo de esmectita muestra un buen desempeño con un $\mathrm{R}^{2}$ de $95 \%$, lo que se refleja además en los gráficos de dispersión presentes en la Figura 12.

\subsection{Cuarzo}

En el modelo de cuarzo muestra un buen desempeño con un $\mathrm{R}^{2}$ de $93 \%$, lo que se refleja además en los gráficos de dispersión presentes en la Figura 13.

\subsection{Ortoclasa}

En el modelo de ortoclasa muestra un buen desempeño con un $\mathrm{R}^{2}$ de $64 \%$, lo que se refleja además en los gráficos de dispersión presentes en la Figura 14.

\subsection{Muscovita}

En el modelo de muscovita muestra un buen desempeño con un $\mathrm{R}^{2}$ de $59 \%$, lo que se refleja además en los gráficos de dispersión presentes en la Figura 15.

\subsection{Caolinita}

En el modelo de caolinita muestra un buen desempeño con un $\mathrm{R}^{2}$ de $62 \%$, lo que se refleja además en los gráficos de dispersión presentes en la Figura 16.

\subsection{Amorfo}

En el modelo de amorfo muestra un buen desempeño con un $\mathrm{R}^{2}$ de $67 \%$, lo que se refleja además en los gráficos de dispersión presentes en la Figura 17.

\subsection{Plagioclasa}

En el modelo de plagioclasa muestra un buen desempeño con un $\mathrm{R}^{2}$ de $78 \%$, teniendo una raíz del error cuadrático

Tabla 4. Resumen de indicador de desempeño para los modelos construidos

\begin{tabular}{|c|c|c|c|c|c|c|}
\hline \multirow{2}{*}{ Especies de Minerales } & \multicolumn{2}{|c|}{ Error cuadrático medio } & \multicolumn{2}{|c|}{ Raiz Error cuadrático medio } & \multicolumn{2}{|c|}{ R cuadrado } \\
\hline & Train & Test & Train & Test & Train & Test \\
\hline Calcita & 1.79 & 2.40 & 1.34 & 1.55 & 0.64 & 0.51 \\
\hline Caolinita & 1.96 & 4.31 & 1.40 & 2.08 & 0.81 & 0.62 \\
\hline Muscovita & 3.15 & 5.86 & 1.78 & 2.42 & 0.75 & 0.59 \\
\hline Plagioclasa (Oligoclasa) & 3.25 & 5.49 & 1.80 & 2.34 & 0.83 & 0.78 \\
\hline Ortoclasa & 4.17 & 5.61 & 2.04 & 2.37 & 0.82 & 0.64 \\
\hline Cuarzo & 6.81 & 13.07 & 2.61 & 3.62 & 0.97 & 0.93 \\
\hline Amorfo & 5.00 & 15.03 & 2.24 & 3.88 & 0.86 & 0.67 \\
\hline Esmectita & 1.01 & 3.36 & 1.01 & 1.83 & 0.99 & 0.95 \\
\hline
\end{tabular}
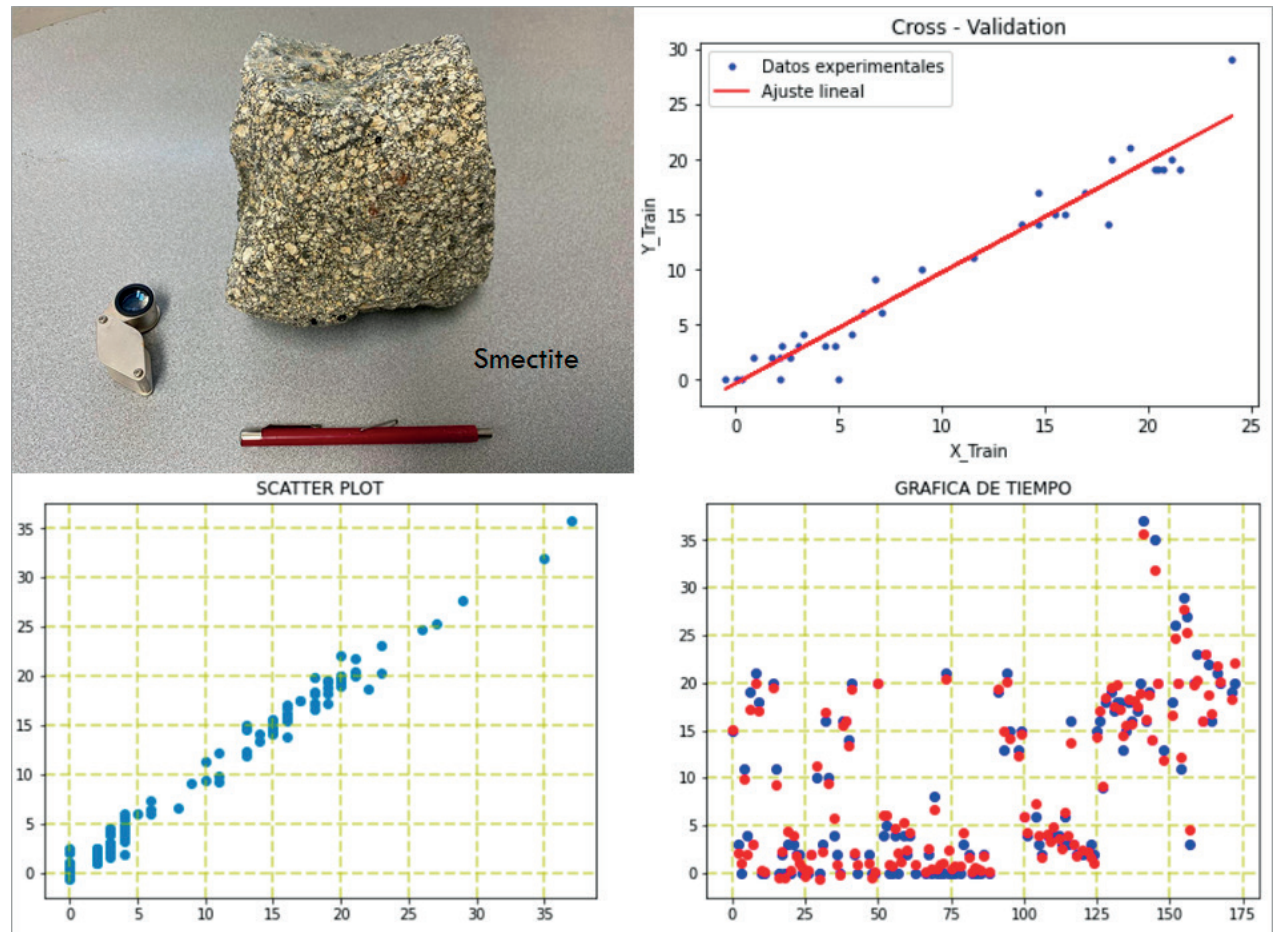

Figura 12. Comportamiento del modelo de Esmectita tanto en etapa de validación cruzada como en la etapa de ajuste. 

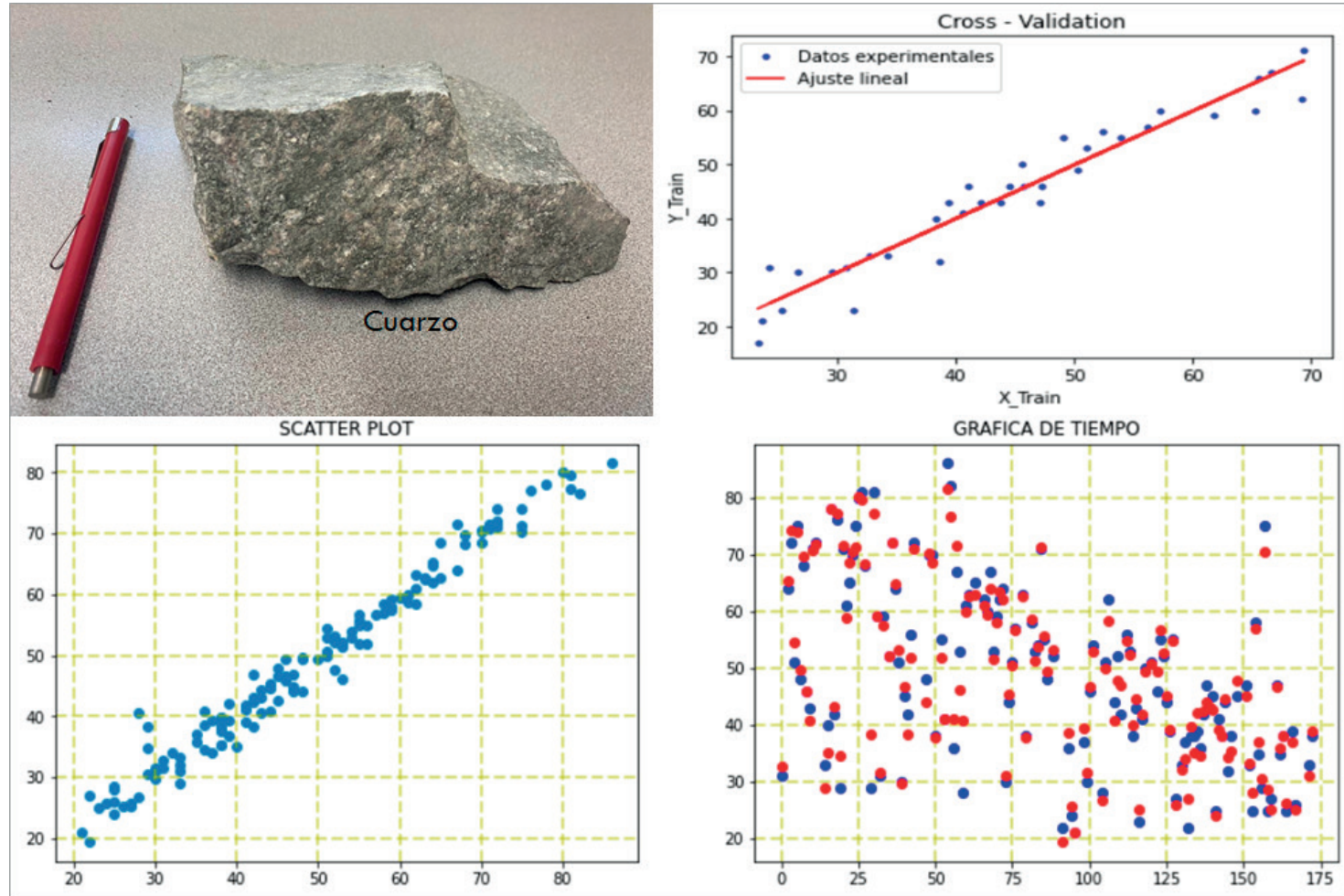

Figura 13. Comportamiento del modelo de Cuarzo tanto en etapa de validación cruzada como en la etapa de ajuste.
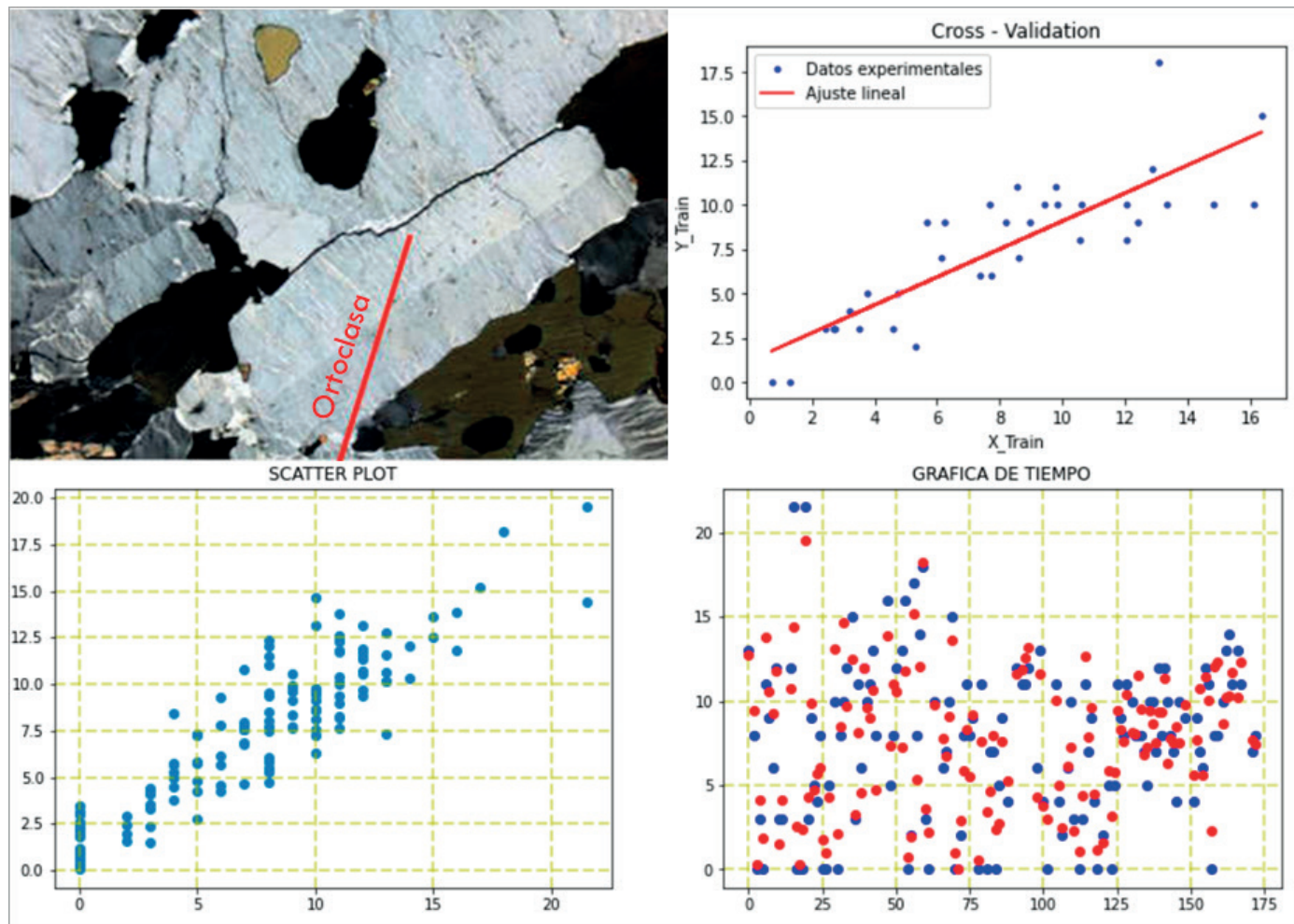

Figura 14. Comportamiento del modelo de Ortoclasa tanto en etapa de validación cruzada como en la etapa de ajuste. 


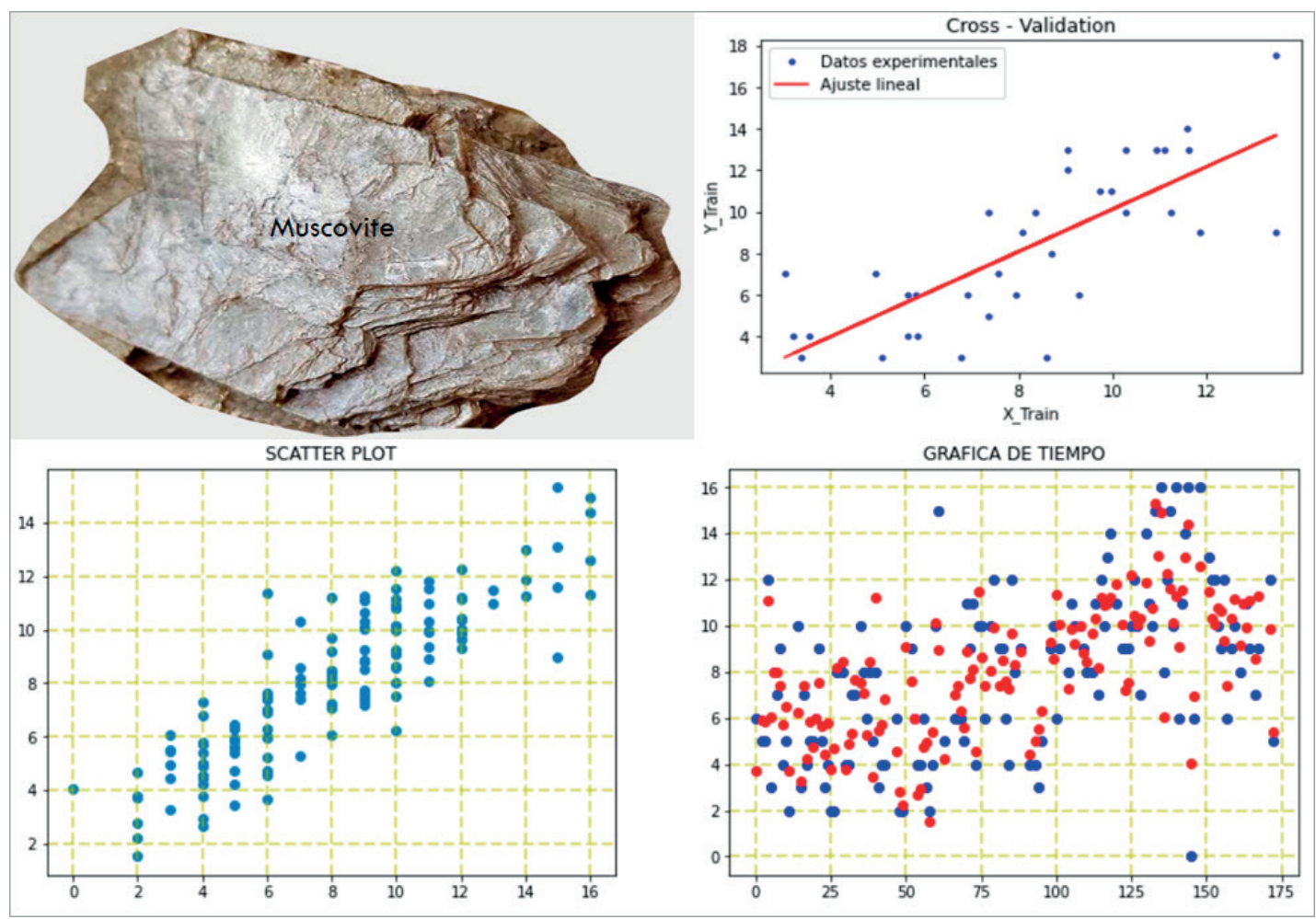

Figura 15. Comportamiento del modelo de Muscovita tanto en etapa de validación cruzada como en la etapa de ajuste.
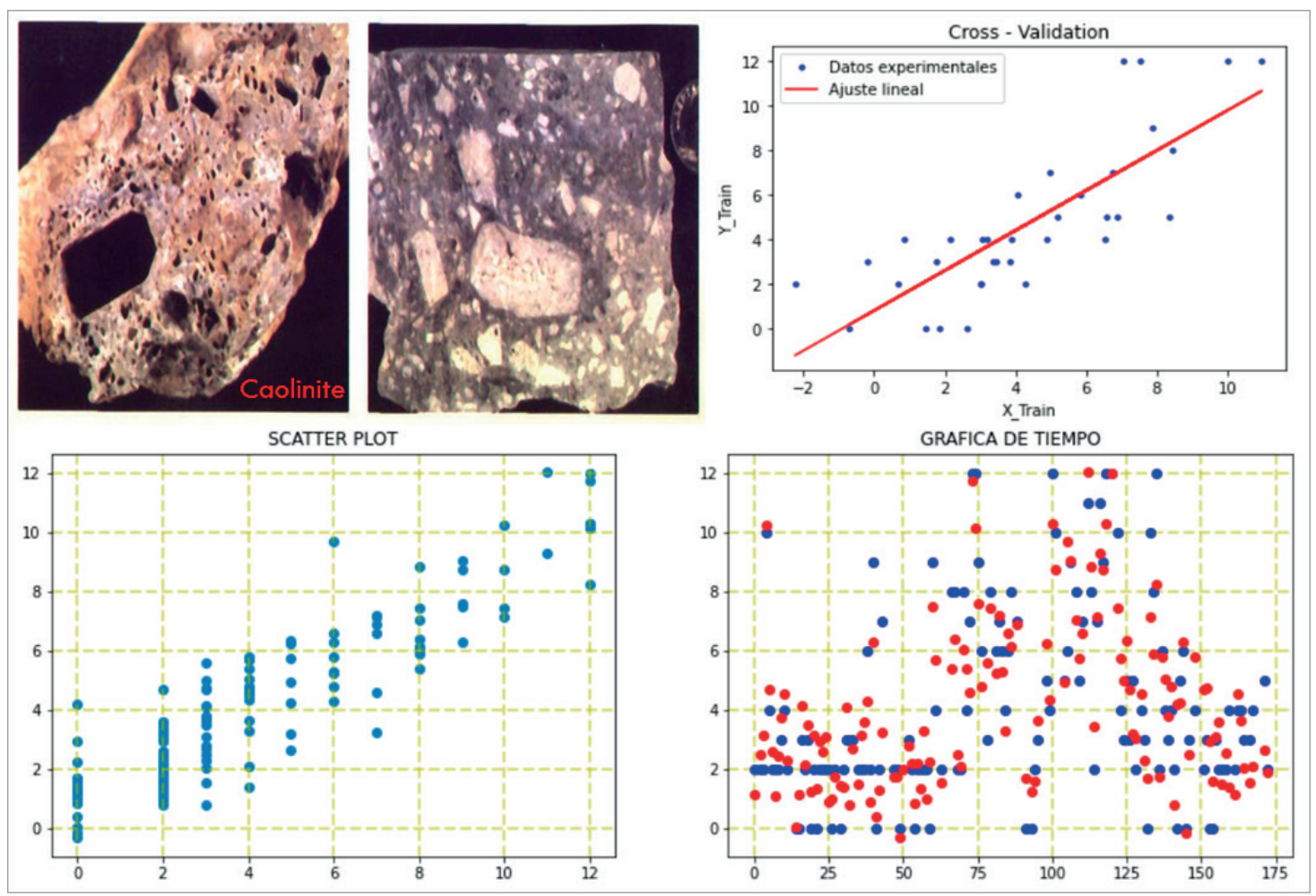

Figura 16. Comportamiento del modelo de Caolinita tanto en etapa de validación cruzada como en la etapa de ajuste. 
medio de $2.34 \%$, lo que se refleja además en los gráficos de dispersión presentes en la Figura 18.

\subsection{Calcita}

En el modelo de calcita muestra un buen desempeño con un $\mathrm{R}^{2}$ de $51 \%$, teniendo una raíz del error cuadrático medio de $1.55 \%$, lo que se refleja además en los gráficos de dispersión presentes en la Figura 19.

\section{DISCUSIÓN}

- El software anaconda navigator permitió generar análisis exploratorio de datos y cálculo estadístico del modelado lineal de ajuste de información significativa. Además de un lenguaje de programación en Python el cual es de uso libre y de bastante robustez para la minería de datos.

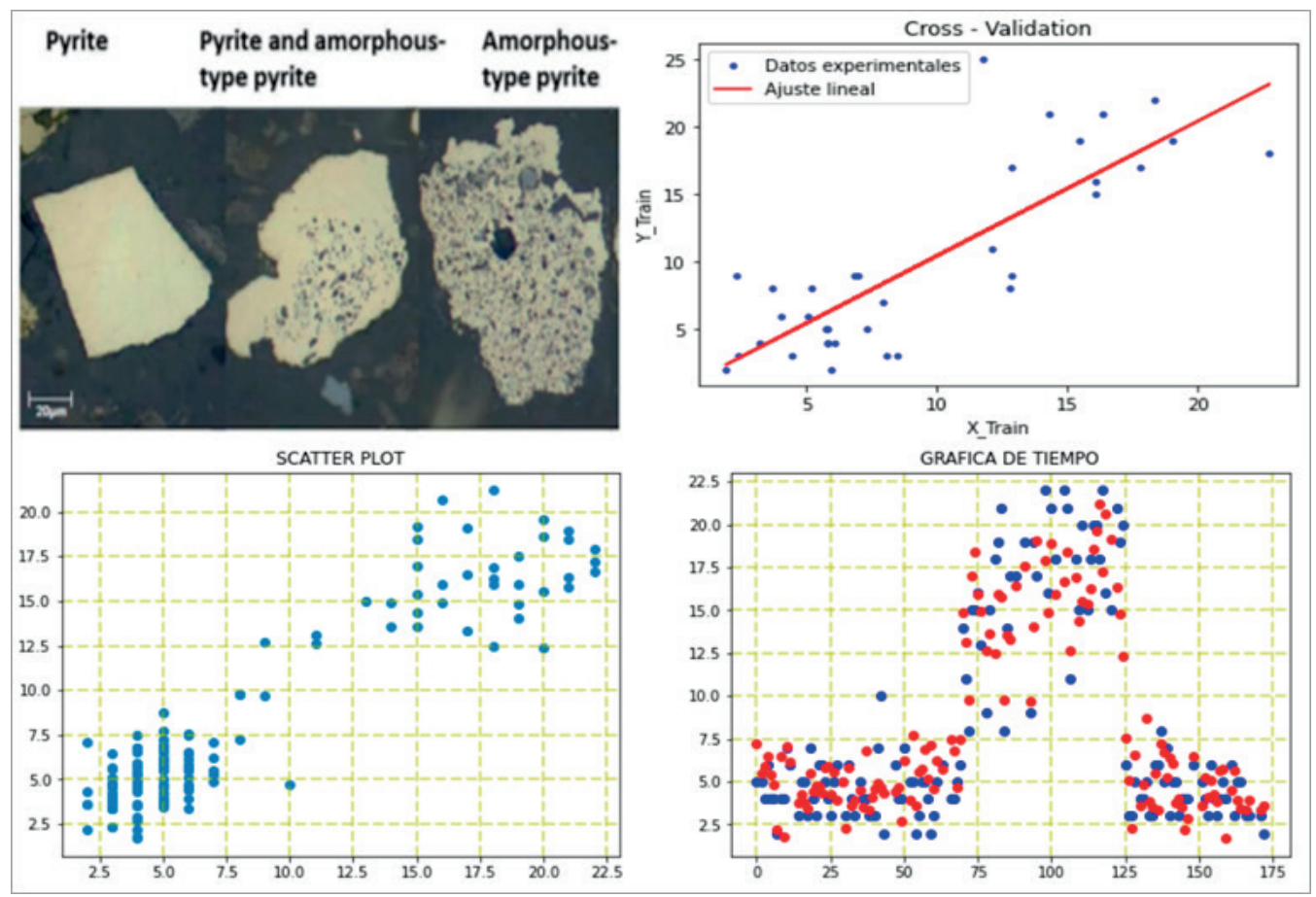

Figura 17. Comportamiento del modelo de Amorfo tanto en etapa de validación cruzada como en la etapa de ajuste.
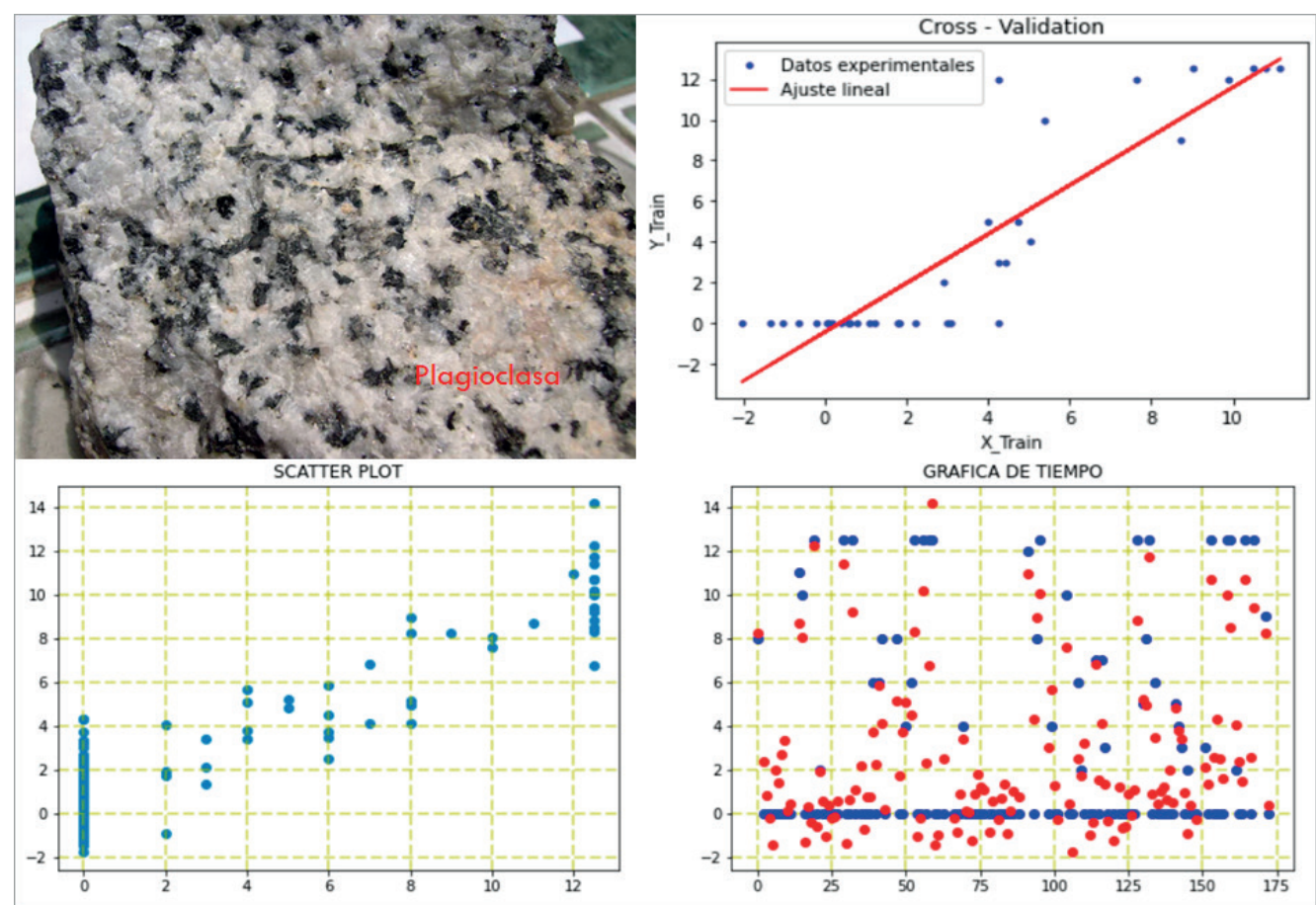

Figura 18. Comportamiento del modelo de Plagioclasa tanto en etapa de validación cruzada como en la etapa de ajuste. 

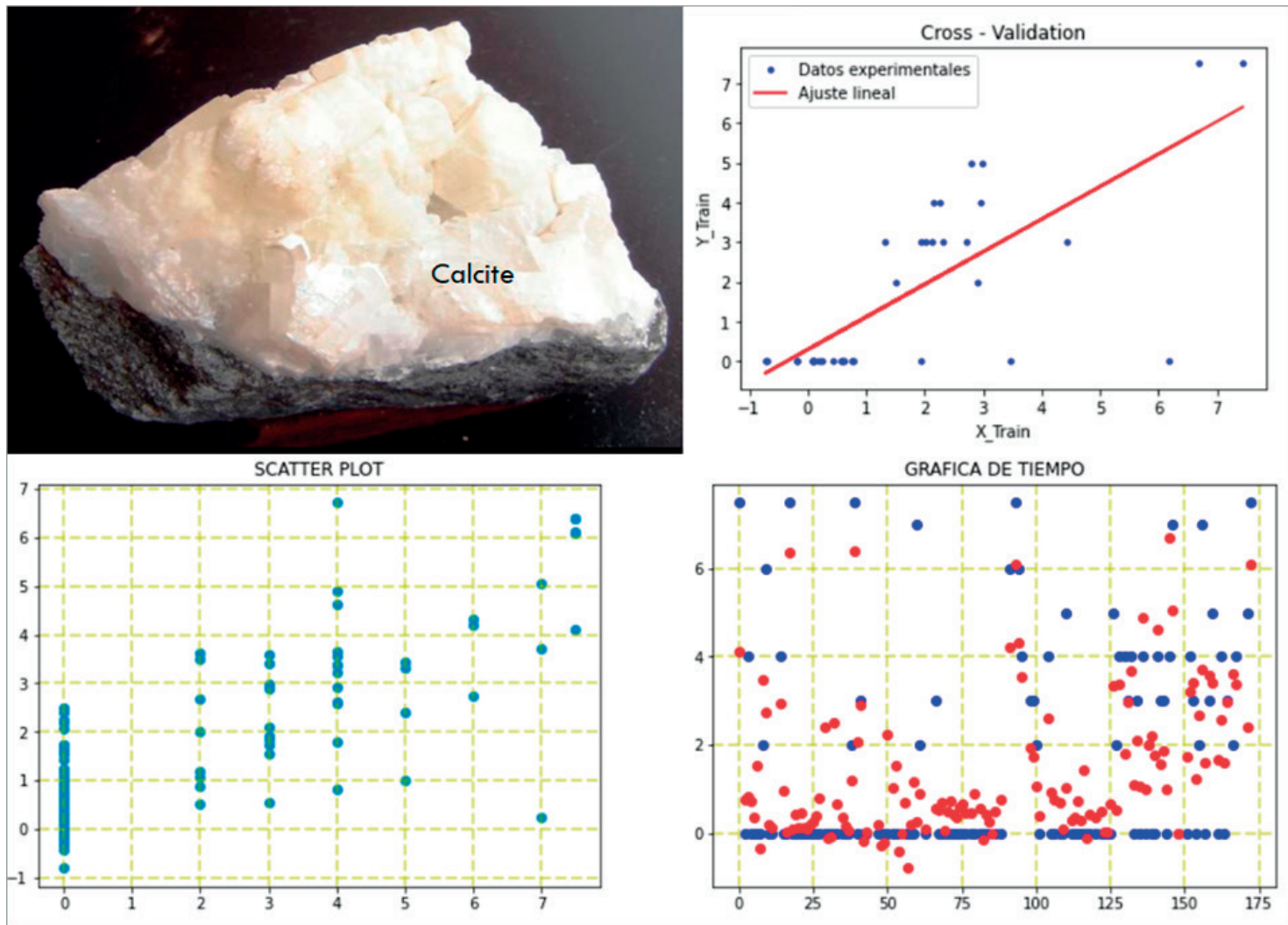

Figura 19. Comportamiento del modelo de Calcita tanto en etapa de validación cruzada como en la etapa de ajuste.

- El generar modelos predictores con una regularización de Ridger, disminuyó de manera significativa la varianza y multicolinialidad, a diferencia del uso tradicional de un método por mínimos cuadrados.

- De acuerdo con el análisis de significancia (Figura 11) se pudo determinar las especies mineralógicas de gangas representativos en los 173 compositos, siendo estos la calcita, caolinita, moscovita, plagioclasas, ortoclasa, cuarzo, amorfo y esméctica.

- Se comprobó la metodología de validación cruzada para encontrar la significa real de los modelos, considerando de este análisis correlaciones significativas para especies como esmectita, cuarzo, ortoclasa, muscovita, caolinita y amorfo. Además, esta metodología pudo determinar que especies de calcitas y plagioclasas a pesar de tener una correlación significativa tenían un alto error cuadrático, lo cual nos indica un sobre ajuste, esto debido a la baja población de datos que se tienen para estas especies debido a la cantidad de compositos generados para el envío de análisis de difracción de rayos $\mathrm{x}$, sin embargo se podría ampliar la cantidad de datos en posteriores campañas de sondajes lo cual generaría un modelo de mayor robustez (Figura 20).

\section{CONCLUSIONES}

- Se pudo demostrar el uso del método alternativo de la espectroscopia de infrarrojo cercano para la determinación cuantitativa de las principales arcillas que afecten a la flotación de espumas. De esta manera integrarse al modelo de bloques de recursos para el planeamiento de minado.

- Los resultados obtenidos indican que la espectroscopía NIR empleando el TERRASPEC 04 se puede usar como una herramienta semi cuantitativa para la determinación rápida y económica de valores de gangas que afectan a la flotación minerales.

\section{AGRADECIMIENTOS}

Al Vicerrectorado de Investigación y Posgrado de la Universidad Nacional Mayor de San Marcos, por el apoyo brindado al Proyecto con Código C18160013, sin financiamiento para la publicación académica del presente artículo científico. 

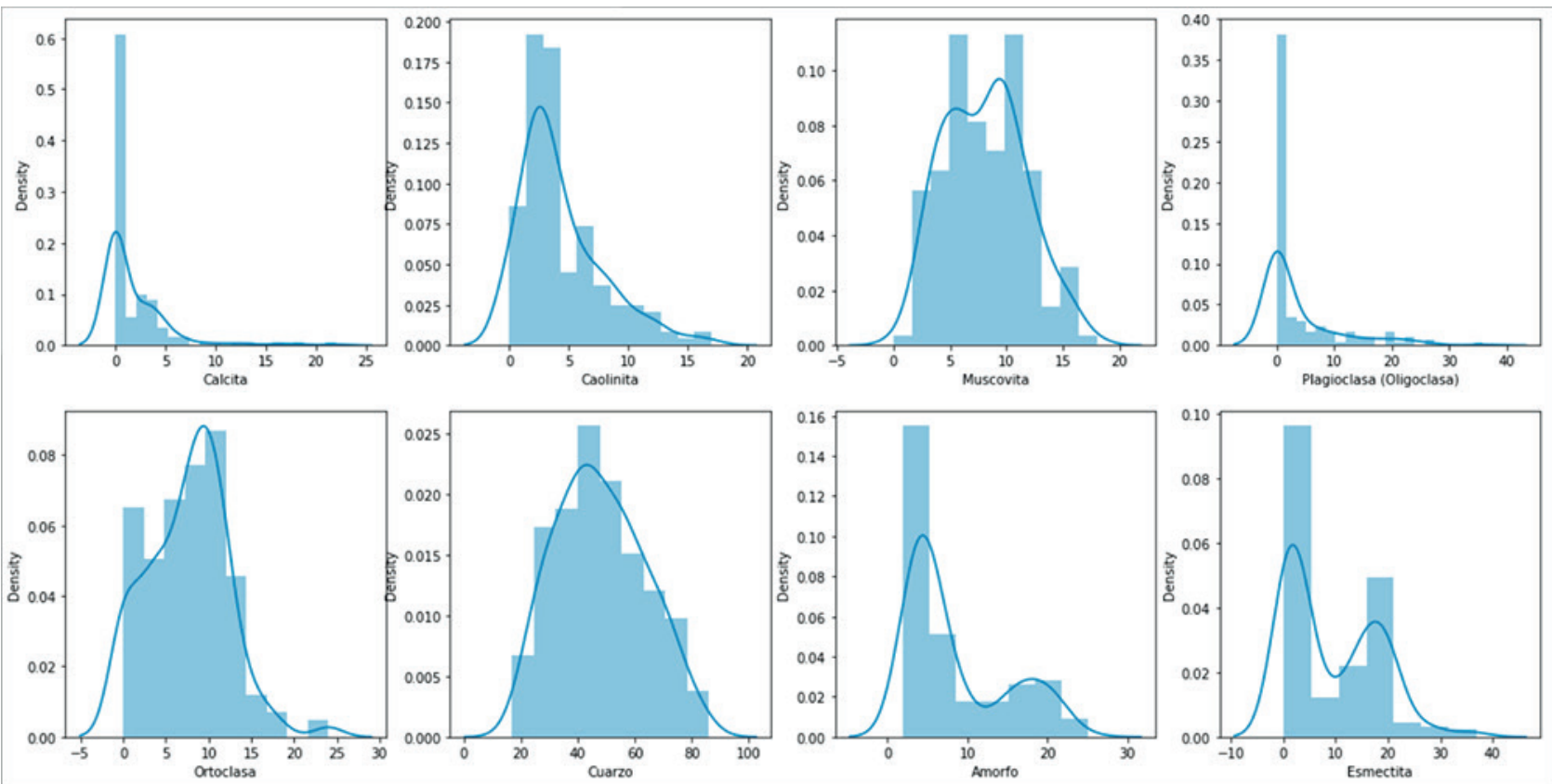

Figura 20. Gráfico de distribución de resultados de ensayos de difracción de rayos $X$ en valores de Z score.

\section{REFERENCIAS}

Canchaya Moya, S. (2008). El modelo geometalúrgico stricto sensu. 4. https://app.ingemmet.gob.pe/biblioteca/pdf/ CPG17-029.pdf

Coleman, R. G. (2000). Prospecting for ophiolites along the California continental margin. Special Paper of the Geological Society of America, 349, 351-364. https://doi. org/10.1130/0-8137-2349-3.351

Gómez Sánchez, P. R. (2015). Correlaciones Entre Espectrometría De Reflectancia, Geoquímica Y Mineralogía De Sedimentos Fluviales De La Cuenca Del Río Elqui, Iv Región, Chile. http://repositorio.uchile.cl/bitstream/handle/2250/133271/ Correlaciones-entre-espectrometria-de-reflectanciageoquimica.pdf? sequence $=1$
González-Páramo, L. M. (2017). Herramientas Avanzadas De Análisis De Datos De Aplicación En Ingeniería Civil. 136. https://repositorio.unican.es/xmlui/bitstream/ handle/10902/12684/Lucia Moreno González-Paramo. pdf? sequence $=1 \&$ isAllowed $=y$

Pallares Cabrera, F. (2014). Desarrollo de un modelo basado en Machine Learning para la predicción. 174. https:// biblioteca.utb.edu.co/notas/tesis/0068209.pdf

Vilches Nuñez, F. E. (2018). Influencia de arcillas en la flotacion de material calcopiritico, proveniente de CODELCO Division Andina [Universidad Técnica Federico Santa María]. https://repositorio.usm.cl/handle/11673/41523 